\title{
Possible Wireless Single-Electron Logic Biased by Electric Field
}

\author{
ALEXANDER N. KOROTKOV \\ Department of Physics, State University of New York, Stony Brook, NY 11794-3800 and Institute of Nuclear Physics, \\ Moscow State University, Moscow 119899, Russia
}

\begin{abstract}
A new type of ultradense logic based on single-electron tunneling is considered. The basic element is a short chain of conducting islands which shows bistable polarization and affects the polarization of neighboring chains. The power is supplied by alternating electric field.
\end{abstract}

Keywords: Single-electron tunneling, Digital circuits, Nanoelectronics

Single-electron effects in systems of small-capacitance tunnel junctions can possibly be used as the physical basis for a new generation of ultradense digital electronics [1]. Two basic types of ultradense single-electron memory and logic have been discussed in the literature. The first way (see Ref. [2] and references therein) is to use single-electron transistors instead of field-effect transistors in digital circuits similar to conventional digital electronics. Another approach [1] is to code information by the presence of an extra electron on a particular conducting island. Both approaches require wires for the power supply and for coupling between elements, and that seems to be inconvenient at the few-nanometer scale. The wires are not required in a suggestion of Ref. [3] (see also Ref. [4]) in which the bistable polarization of the basic element and the local interactions between neighboring elements are used. In this approach there is no power supply, and the only driving force is the fixed polarization of "edge" elements (so-called "ground state computing"). The difficulties of "ground state computing" originate from the fact that even for a large number of elements in a circuit the total energy gain is only of the order of one element- element interaction energy. To provide the sequential switching of elements, this energy gain should be distributed among all switching events. This requires complicated design, leads to very low parameter margins, and allows only small circuits.

The single-electron logic considered in the present paper (see also Ref. [5]) combines the good features of suggestions mentioned above. Similar to systems suggested in Refs. [3,4] our "device" does not have wires. However, we use the traditional dissipative way of logical computation which provides reliability and allows arbitrary large number of elements in a device. The power is supplied by the changes of external electric field leading to a nonequilibrium state.

A chain of closely located conducting islands (see Figs. 1 and 2) serves as the basic element of the device. Small "puddles" of 2D electron gas, small metallic droplets on an insulating substrate, or conducting clusters in a dielectric matrix are possible implementations of the islands.

Application of an in-plane electric field $E$ creates the voltage between the islands. When $E$ exceeds the Coulomb blockade threshold $E_{t}$, tunneling occurs 
somewhere inside the chain. The external field drags the electron-hole pair towards the edges of the chain, creating the polarized state. If we now decrease the field $E$, than the pair eventually annihilate, however, it will occur at the field $E_{a}$ considerably smaller than $E_{t}$. Stability of both polarized and nonpolarized states for $E$ between $E_{a}$ and $E_{t}$ allows to use these states as logical zero and unity.

The polarization change can propagate along a line of closely located chains (Fig. 1a). Suppose that all chains are not polarized initially, and $E$ is slightly less than $E_{t}$. This is a metastable state. If one chain becomes polarized, the field of extra electron (hole) on the edge island increases the potential difference between neighboring islands of the next chain (Fig. 1a). This makes tunneling energetically favorable and leads to polarization of the next chain (electrons do not tunnel between chains because of the larger distance). This in turn polarizes the next chain and so on. The unidirectional propagation (from left to right in Fig. 1a) is a consequence of the asymmetry of the circuit.

The natural fan-out of the signal into two lines can be realized if both edge islands of a chain are used to trigger the next chains (Fig. 1b).
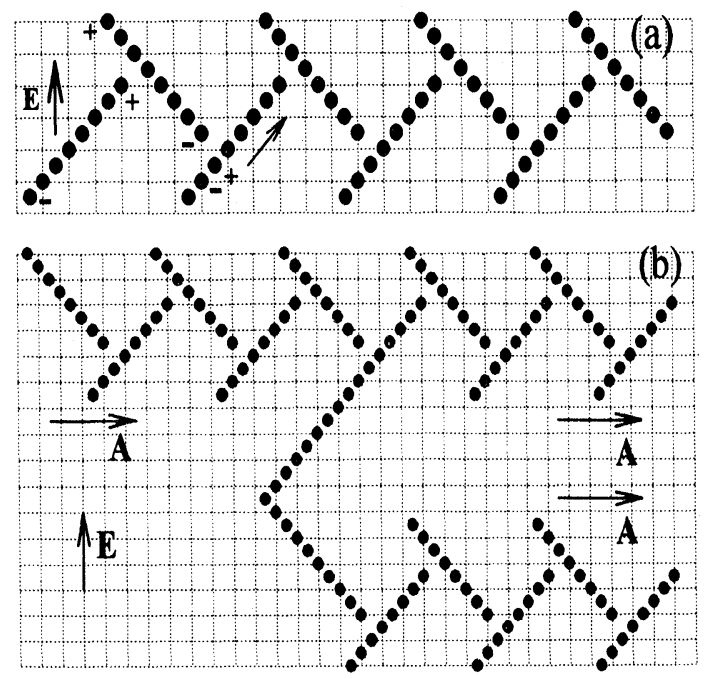

FIGURE 1 (a) The line of chains propagating the signal in the direction (from left to right) perpendicular to the external field $E$. (b) The circuit for the fan-out of signal A
A "bi-controlled" chain (fifth from the right in Fig. 2a) which can be triggered by the polarization of either of two neighboring input chains can be used as the basic part of the logical gate OR.

The logical gate AND can be designed similar to the OR gate, but with slightly larger distance between the "bi-controlled" chain and the neighboring input chains, in order to decrease their influence. Another possibility is to make the islands of "bi-controlled" chain slightly smaller in order to increase the Coulomb blockade energy.

Because of the asymmetry between logical zero and unity the design of the inverter (NOT gate) is relatively complex. The circuit shown in Fig. $2 b$ implements the logical function (NOT A).AND.B if the signal from input $\mathrm{A}$ comes before the signal from input $\mathrm{B}$. The signal $\mathrm{B}$ will propagate to the output as in the usual propagation line, unless the chains of input A are polarized. This circuit can be used as NOT A, if logical unity always comes from input B and it comes later than signal A.

The dynamics of circuits shown in Figs. 1 and 2 was simulated numerically using a standard MonteCarlo approach based on the "orthodox" theory [1] of

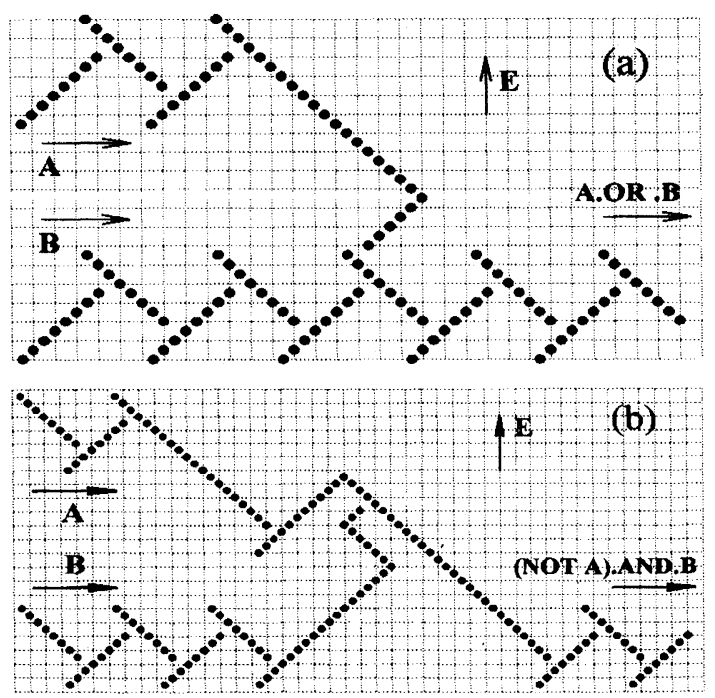

FIGURE 2 (a) The logical gate OR. The chain fifth from the right can be triggered by either signal A or B. Similar layout can operate as AND gate. (b) The circuit which operates as (NOT A).AND.B if the signal A comes before signal B 
single-electron tunneling (resistance $R$ between neighboring islands was assumed to be much larger than $R_{Q}=\pi \hbar / 2 e^{2}$ ). The capacitance matrix for spherical islands was calculated numerically using the method of multiple electrostatic images. For each initial charge the positions and magnitudes of image charges in all islands were calculated, then for each image all images of the next generation were calculated, and so on until the desired accuracy is reached (typically, 10-20 iterations were necessary). Because of the large number of islands in a simulated circuit (typically, more than 100), the number of images grows very fast. To solve this problem, only the total charge and the total dipole momentum of sufficiently small image charges inside a sphere were stored.

The distance between centers of neighboring islands in a chain was taken to be $\sqrt{2}$ times larger than the island diameter. Background charges were assumed to be zero.

According to the results of simulation, the correct operation of all gates requires that the magnitude of external field $E$ lies within 5\% margin [5]. This number gives also a crude estimate of the margins for other parameters (fluctuations of radii, spacings, etc.)

Considered logical gates together with propagation lines and fan-out circuits, are sufficient for computing. In the simplest mode of operation, all chains inside a device have zero polarization and external field is zero in the initial state. Then external field increases up to a value for which all gates operate correctly, and elements start to switch in accordance with the input information flowing from the edges of the device. The result of the computation is the final polarization of output elements which can be read out, for example, by single-electron transistors. This simplest mode of operation can obviously be improved by the use of periodic changes of the external field ("clock cycles"). Properly chosen levels of the field can reset some elements but preserve the information in other elements.

The work was supported in part by ONR, AFOSR, and Russian RFFR grants.

\section{References}

[1] D. V. Averin and K. K. Likharev, "Possible Applications of the Single Charge Tunneling", in: Single Charge Tunneling, ed. by H. Grabert and M. Devoret (Plenum, New York, 1992), pp. 311-332.

[2] A. N. Korotkov, R. H. Chen, and K. K. Likharev, "Possible Performance of Single-Electron Transistors in Digital Circuits", J. Appl. Phys., vol. 78, pp. 2520-2530, 1995.

[3] C. S. Lent, P. D. Tougaw, W. Porod, and G. H. Bernstein, "Quantum Cellular Automata", Nanotechnology, vol. 4, pp. 49-57, 1993.

[4] S. Bandyopadhyay, B. Das, and A. E. Miller, "Supercomputing with Spin-Polarized Single Electrons in a Quantum Coupled Architecture", Nanotechnology, vol. 5, pp. 113-133, 1994.

[5] A. N. Korotkov, "Wireless Single-Electron Logic", Appl. Phys. Lett., vol. 67, pp. 2412-2414, 1995.

\section{Biography}

Alexander Korotkov (e-mail: akorotkov@ccmail. sunysb.edu) is currently a Research Scientist in the Dept. of Physics at the State University of New York at Stony Brook. His research interests include physics and possible applications of single-electron tunneling and transport properties of semiconductor heterostructures. 

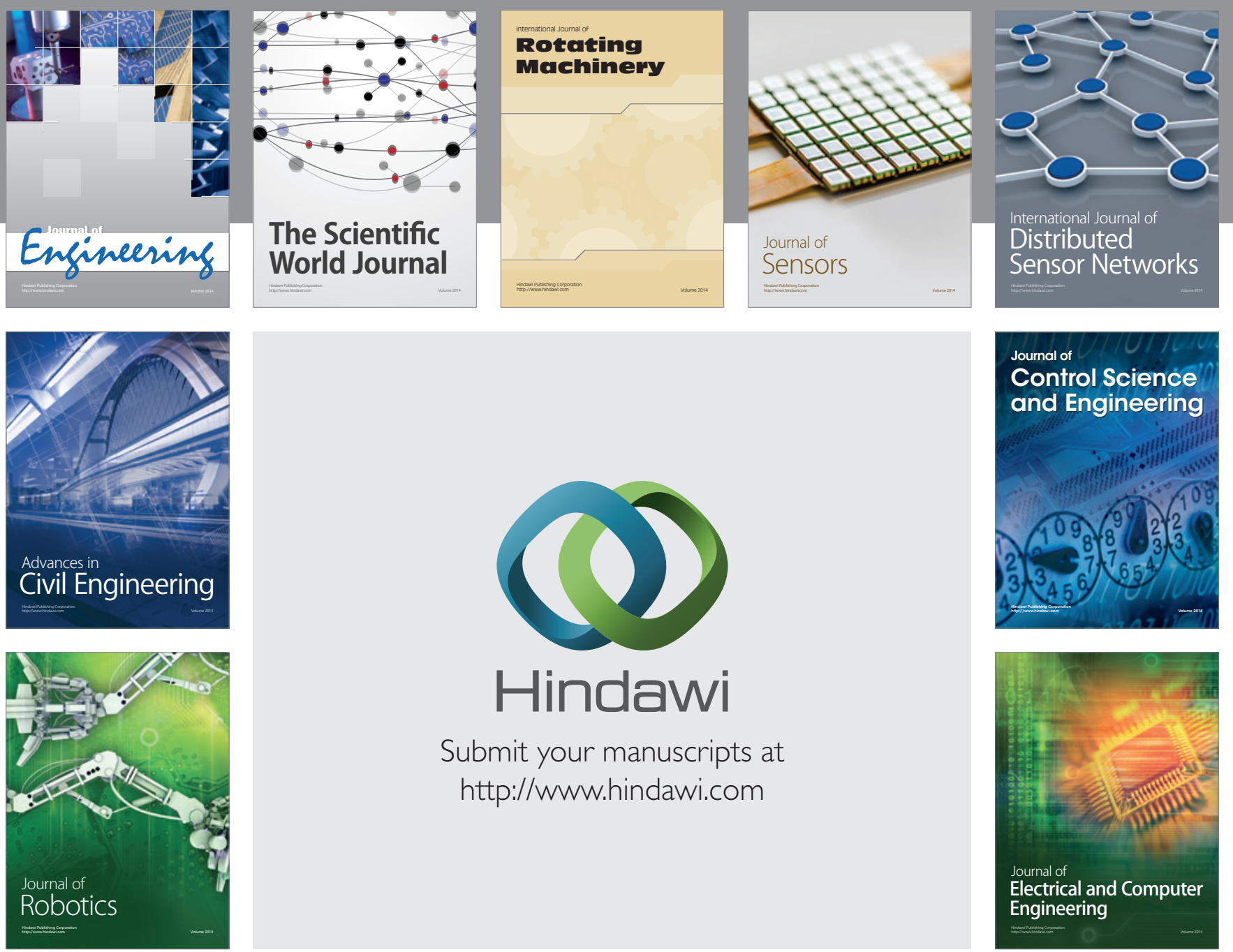

Submit your manuscripts at

http://www.hindawi.com
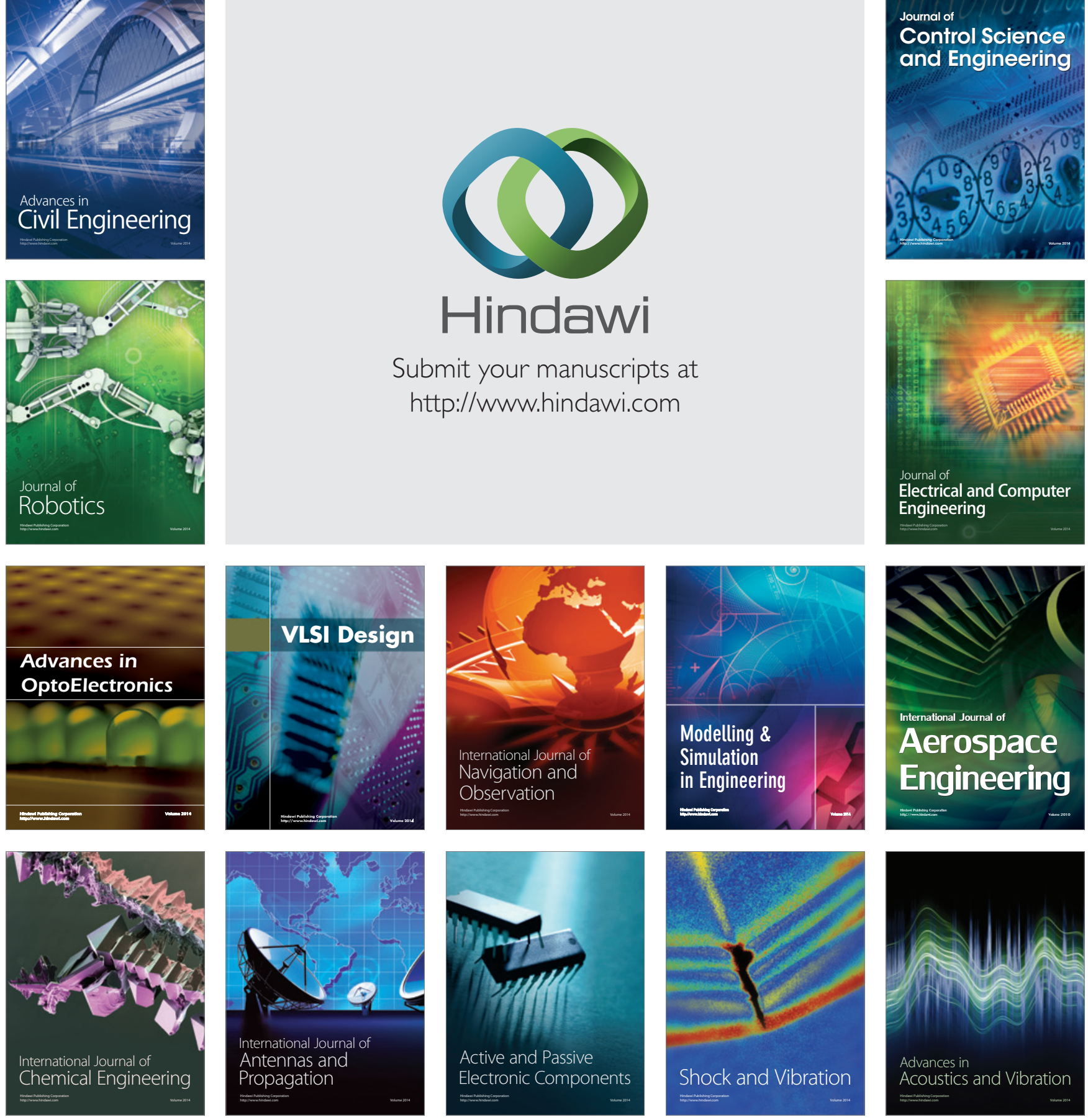